\title{
Impact of Low Back Pain on Quality of Life: Assessment by Patient Functionality Questionnaire and Treatment Results Using a Combination of Diclofenac plus B Vitamins or Diclofenac Monotherapy
}

\author{
Mauro Geller ${ }^{*}$, Marco Antonio Mibielli' ${ }^{1}$ Carlos Pereira Nunes ${ }^{1}$, \\ Adenilson de Souza da Fonseca1, Stephanie Wrobel Goldberg' ${ }^{2}$, Lisa Oliveira ${ }^{1}$ \\ ${ }^{1}$ Teresópolis Medical School, UNIFESO, Rio de Janeiro, Brazil \\ ${ }^{2}$ Washington University School of Medicine, St. Louis, MO, USA \\ Email: "maurogeller@gmail.com
}

Received 12 November 2015; accepted 13 February 2016; published 17 February 2016

Copyright (C) 2016 by authors and Scientific Research Publishing Inc.

This work is licensed under the Creative Commons Attribution International License (CC BY). http://creativecommons.org/licenses/by/4.0/

\section{(c) (i) Open Access}

\section{Abstract}

Objective: To analyze the Patient Functionality Questionnaire (PFQ) scores of the patients treated with either Diclofenac + B vitamins (Group DB) or Diclofenac monotherapy (Group D) in order to better understand the impact of the combination of diclofenac with vitamins $B_{1}, B_{6}$, and $B_{12}$ on quality of life. Research Design/Methods: We performed a post-hoc analysis of tabulated data generated during the DOLOR study (Diclofenac plus B vitamins versus diclofenac monotherapy in lumbago: the DOLOR study), using the software GraphPad Prism 5 for Windows, v5.04. The absolute number or percentage of "True"/"False" respondents for each questionnaire item at each study visit, together with the absolute number and percentage of subjects with no change, improvement, or worsening of each $\mathrm{PFQ}$ item at each study visit, within and between treatment groups were arranged on contingency tables and analyzed with the Chi-squared or Fisher's Exact test. Results: At pretreatment there were no statistically significant differences between treatment groups ( $p>0.05$ for all items). At Visit 2 in both treatment groups, there were statistically significant improvements in individual question responses in each of the 12 items of the questionnaire ( $p<0.05$ for all items). At Visit 2, comparing the distribution of patients in each treatment group with "improvement", "no change", or "worsening", there was a statistically significant superiority among subjects of group DB in response to items $1,6,8$, and 10. At Visit 3, both groups had statistically significant $(p<0.001)$ improvements in each individual item of the $P F Q$ in relation to pre-

\footnotetext{
*Corresponding author.
}

How to cite this paper: Geller, M., et al. (2016) Impact of Low Back Pain on Quality of Life: Assessment by Patient Functionality Questionnaire and Treatment Results Using a Combination of Diclofenac plus B Vitamins or Diclofenac Monotherapy. International Journal of Clinical Medicine, 7, 113-119. http://dx.doi.org/10.4236/ijcm.2016.72012 
treatment values. The distribution of subjects in each treatment group presenting with "improvement", "no change", or "worsening" at Visit 3 varied significantly in favor of group DB in the responses to item 4. At Visit 4, the subjects remaining in treatment in both groups showed statistically significant improvement in PFQ responses in relation to pretreatment values $(p<0.05)$. The distribution of subjects in each treatment group presenting with "improvement" or "no change" at Visit 4 varied significantly in favor of group $D$ in the responses to items 5, 8, and 12. Conclusion: Improvement in overall PFQ scores was observed in both treatment groups, though the specific items of the questionnaire of the subjects in group DB at Visit 2 showed greater improvement in areas related to sleep quality, mobility, ability to wash and dry, ability to walk distances, and posture comfort. These results serve to corroborate the previously published data, which indicates a benefit of combining the $B$ vitamins with diclofenac in the treatment of patients with low back pain, yielding shorter treatment time to resolution of the lumbago in the treated patients.

\section{Keywords}

Low Back Pain, Quality Of Life, Diclofenac, Thiamine, Pyridoxine, Cyanocobalamin

\section{Introduction}

In a previous clinical trial, we evaluated and compared the action of the combination of diclofenac $(50 \mathrm{mg})$ and vitamins $\mathrm{B}_{1}$ (thiamine mononitrate, $50 \mathrm{mg}$ ), $\mathrm{B}_{6}$ (pyridoxine hydrochloride, $50 \mathrm{mg}$ ), and $\mathrm{B}_{12}$ (cyanocobalamin, 1 $\mathrm{mg}$ ) versus diclofenac $(50 \mathrm{mg})$ monotherapy among patients presenting with acute low back pain. Among the outcome measures evaluated was the Patient Functionality Questionnaire, a 12-item questionnaire assessing the impact of low back pain on patient's quality of life in specific areas, answered by the patient at each study visit with True/False. One point was awarded for each "true" answer and the total scores of each questionnaire were reported in the previously published paper entitled "Diclofenac plus B vitamins versus diclofenac monotherapy in lumbago: the DOLOR study" [1].

However, we chose to take a closer look at the results of the PFQ scores in each treatment group (Group DB: Diclofenac + B vitamins and Group D: Diclofenac monotherapy) and at each study visit, in order to better understand the impact of the combination of diclofenac with vitamins $\mathrm{B}_{1}, \mathrm{~B}_{6}$, and $\mathrm{B}_{12}$ on quality of life. Study visits occurred at Visit 1-pretreatment, Visit 2-following 3 days of treatment, Visit 3-following 5 days of treatment, and Visit 4-at the end of the 7-day treatment period. The PFQ in its entirety is reproduced below; the subjects answered either "true" or "false" to each question:

Due to my back pain:

1) I do not sleep well;

2) I have to lie down more often;

3) It is difficult for me to get up from my bed or a chair;

4) I can stand only for a short while;

5) I can walk up stairs only slowly;

6) It is difficult for me to wash or dry off my whole body;

7) It is difficult for me to put on my clothes;

8) I can only walk short distances;

9) I try to avoid picking things up from the floor;

10) I have to change my posture more often;

11) I cannot carry heavy things;

12) I have to ask other people for assistance.

It is important to note that in the DOLOR study, patients presenting with significant clinical improvement at study Visits 2 and 3 were allowed to exit the study prematurely. This improvement was defined as Visual Ana$\log$ Pain Scale (VAS) scores equal to or less than $20 \mathrm{~mm}$ on a $100 \mathrm{~mm}$ scale, and patient satisfaction with pain reduction. Accordingly, at Visit 2, 87 patients exited the study due to treatment success after 3 days in the DB group and 55 patients exited the study in the D group, leaving 87 patients continuing treatment in group DB and 
120 patients in Group D. At Visit 3, after 5 days, a further 71 patients exited the study in group DB and 52 in group D, leaving 16 subjects continuing treatment in group DB and 68 subjects in group D.

\section{Material \& Methods}

We performed a post-hoc analysis of previously tabulated data from the Clinical Research Forms of the DOLOR study, using the software GraphPad Prism 5 for Windows, version 5.04, Graph Pad Software, San Diego, California, USA. The absolute number or percentage of "True"/“False" respondents for each questionnaire item, at each study visit, within and between treatment groups were arranged on contingency tables and analyzed with the Chi-squared or Fisher's Exact test. Statistical significance was defined with a two-tailed p value of less than 0.05 with a confidence interval of $95 \%$. Additionally, the absolute number and percentage of patients with no change, improvement, or worsening of each individual item of the PFQ at each study visit was determined by numerically coding "True"/“False" responses and obtaining the differences between Visits 2, 3, and 4 and pretreatment values. These results were also arranged on contingency tables and analyzed with the Chi-squared test.

\section{Results}

At Visit 1 (pretreatment), total mean PFQ scores were 9 and 9 in groups DB and D, respectively; there were no statistically significant differences between treatment groups in responses in any of the individual questionnaire items ( $p>0.05$ for all items). At Visit 2, mean PFQ in group DB was 4.59 while that of group D was 5.8. The responses to individual items in each group at Visits 1 and 2 can be visualized in Figure 1(a) and Figure 1(b). The most drastic changes in PFQ responses occurred at Visit 2 in both treatment groups, with statistically significant improvements in individual question responses in each of the 12 items of the questionnaire observed in both treatment groups ( $\mathrm{p}<0.05$ for all items).

At Visit 2, comparing the distribution of patients in each treatment group with "improvement", "no change", or "worsening", there was a statistically significant superiority among subjects of group DB in response to items $1\left(\chi^{2}=12.64 ; \mathrm{df}=2 ; \mathrm{p}=0.0018\right) ; 3\left(\chi^{2}=9.54 ; \mathrm{df}=2 ; \mathrm{p}=0.0085\right) ; 6\left(\chi^{2}=12.66 ; \mathrm{df}=2 ; \mathrm{p}=0.0018\right) ; 8\left(\chi^{2}=6.99\right.$; $\mathrm{df}=2 ; \mathrm{p}=0.03)$; and $10\left(\chi^{2}=6.95 ; \mathrm{df}=2 ; \mathrm{p}=0.031\right)$. None of the items responded showed statistically significant superiority in favor of group D at Visit 2 (Figure 2).

At Visit 3, among subjects of Group DB, there were statistically significant $(\mathrm{p}<0.001)$ improvements in each individual item of the PFQ in relation to pretreatment values. The same was true among the subjects of Group D. The distribution of subjects in each treatment group presenting with "improvement", "no change", or "worsening” at Visit 3 varied significantly in favor of group DB in the responses to item $4\left(\chi^{2}=6.065\right.$; $\mathrm{df}=2$; $\left.\mathrm{p}=0.048\right)$; no other statistically significant difference between groups was noted at Visit 3 (Figure 3).

At Visit 4, the subjects remaining in treatment in both groups showed statistically significant improvement in PFQ responses in relation to pretreatment values ( $\mathrm{p}<0.05$ for all responses, in both treatment groups). There were no subjects in either treatment group presenting with "worsening" at Visit 4 in relation to pretreatment responses. The distribution of subjects in each treatment group presenting with "improvement", "no change", or "worsening" at Visit 4 varied significantly in favor of group D in the responses to items $5(\mathrm{p}<0.0001), 8(\mathrm{p}<$ $0.0001)$ and $12(\mathrm{p}=0.042)$.

\section{Discussion}

In this analysis, we investigated the individual "true"/“false” responses to the 12-item PFQ recorded during the DOLOR study, for the purpose of better understanding the impact of the addition of the B vitamins to diclofenac therapy of low back pain on the PFQ. The most prominent improvement among study participants occurred at Visit 2 among the subjects treated with the combination of diclofenac and the B vitamins, specifically in areas related to sleep quality, mobility, ability to wash and dry, ability to walk distances, and posture comfort. On the other hand, subjects treated solely with diclofenac took a longer time but showed improvements at treatment days 5 and 7 (Visits 3 and 4).

While widely used and somewhat ubiquitous, in the healthcare setting, the term quality of life is used to describe the perceived quality of an individual patient's daily life, taking into account physical, functional, emotional, and social elements. In painful conditions, evaluation of quality of life is an important part of a range of tools used in assessing treatment effectiveness of a given intervention. Quality of life is especially important 


\section{Group DB: PFQ Scores at Visit 1 and Visit 2}

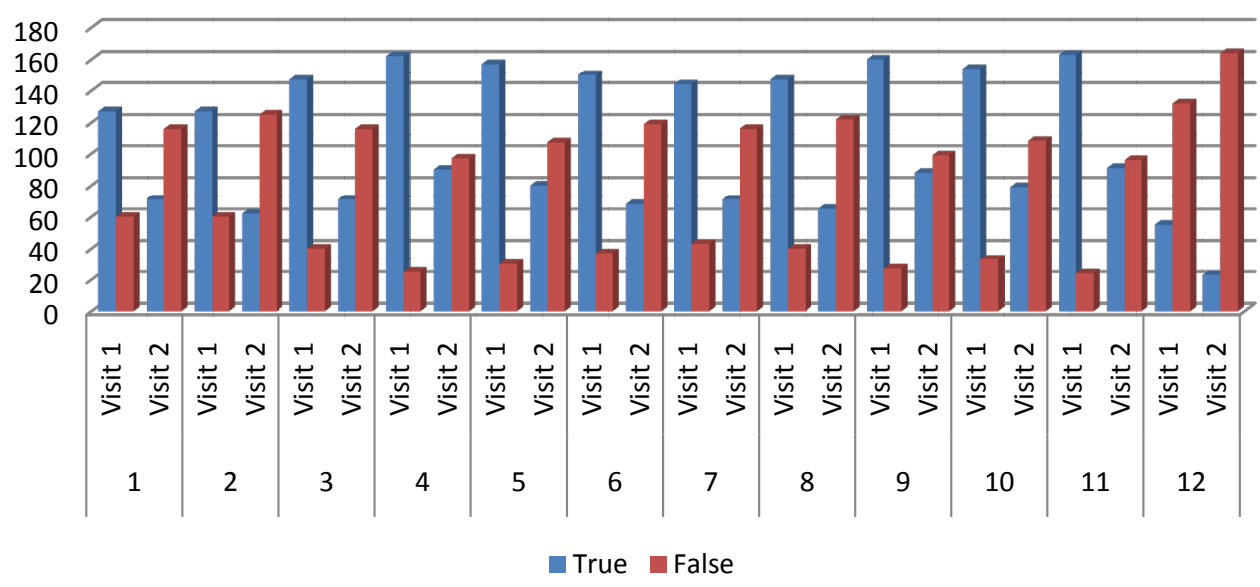

\begin{tabular}{|ll|ll|}
\hline 1 & I do not sleep well & 2 & I have to lie down more often \\
3 & It is difficult for me to get up from my bed or a chair & 4 & I can stand only for a short while \\
5 & I can walk up stairs only slowly & 6 & It is difficult for me to wash or dry off my whole body \\
7 & It is difficult for me to put on my clothes & 8 & I can only walk short distances \\
9 & I try to avoid picking things up from the floor & 10 & I have to change my posture more often \\
11 & I cannot carry heavy things & 12 & I have to ask other people for assistance \\
\hline
\end{tabular}

(a)

\section{Group D: PFQ Scores at Visit 1 and Visit 2}

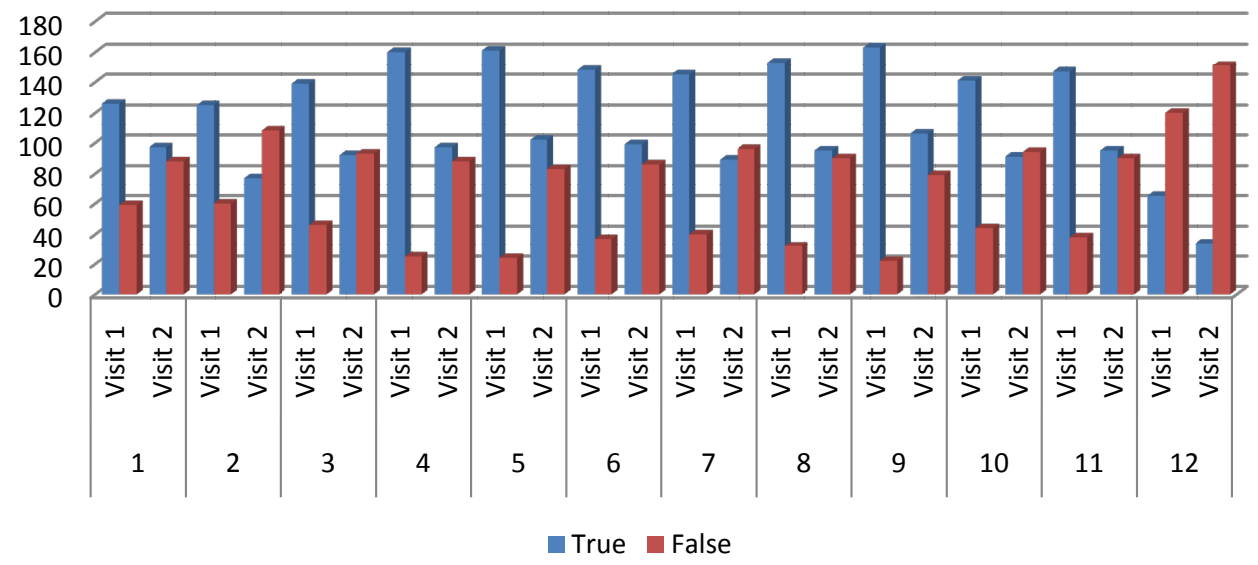

\begin{tabular}{|ll|ll|}
\hline 1 & I do not sleep well & 2 & I have to lie down more often \\
3 & It is difficult for me to get up from my bed or a chair & 4 & I can stand only for a short while \\
5 & I can walk up stairs only slowly & 6 & It is difficult for me to wash or dry off my whole body \\
7 & It is difficult for me to put on my clothes & 8 & I can only walk short distances \\
9 & I try to avoid picking things up from the floor & 10 & I have to change my posture more often \\
11 & I cannot carry heavy things & 12 & I have to ask other people for assistance \\
\hline
\end{tabular}

(b)

Figure 1. a): PFQ responses at Visit 1 and Visit 2 among patients in the DB treatment group; b): PFQ responses at Visit 1 and Visit 2 among patients in the D treatment group.

when discussing low back pain, both due to the prevalence of the condition and because of its influence on various areas of daily life [2]-[4].

Low back pain is an extremely common but very important health condition, affecting up to $80 \%$ of all adults at some point in time [4]. It represents the second most common reason for symptom-driven patient visits to the 


\section{PFQ Improvement at Visit 2}

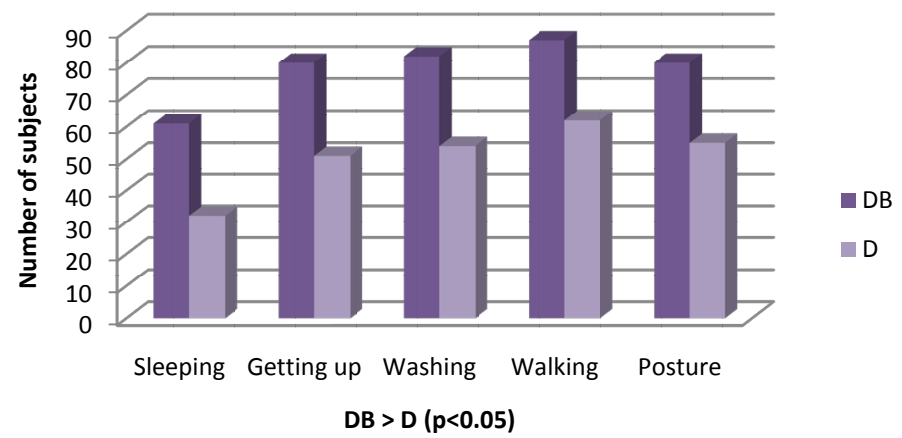

Figure 2. PFQ Responses at Visit 2 with statistically superior results in Group DB compared to Group D.

\section{Visit 3: Percentage of Responders in Relation to Pretreatment}

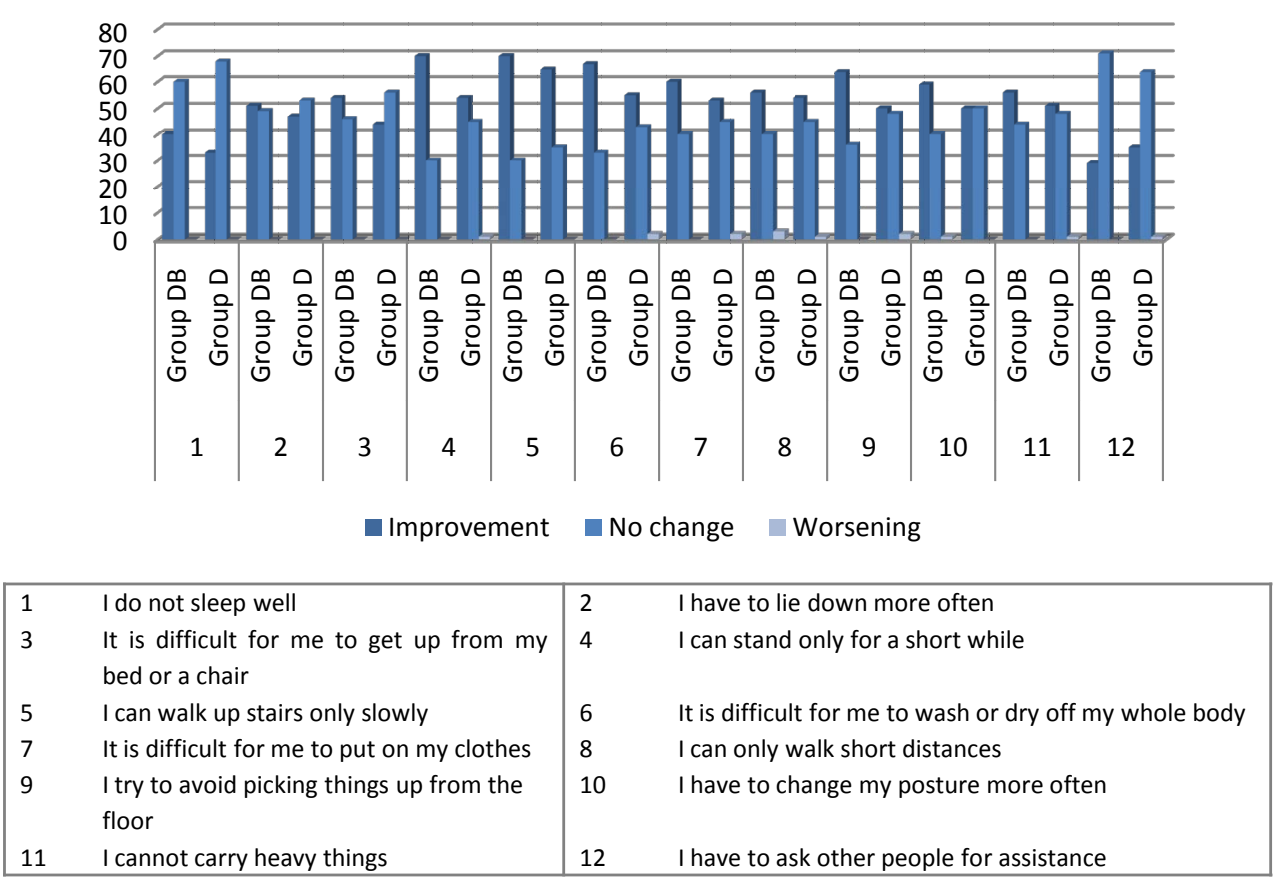

Figure 3. PFQ results at Visit 3 in relation to pretreatment results.

doctor's office after the common cold [5]. Low back pain carries an influence on various areas of daily life, including ability to work, exercise, and perform domestic activities. There is a considerable economic burden associated with the condition, for reasons ranging from its place as the most common cause of work-related disability and a frequent cause of missed work days, to direct costs of diagnosis and treatment [6] [7].

The correlation between sleep quality and low back pain has been investigated in studies of patients with chronic low back pain. Identified aspects of sleep negatively impacted by the presence of low back pain include: greater sleep disturbance, shorter sleep duration, reduction in sleep quality, negative impact on daytime function, increased sleep dissatisfaction and distress, and reduced ability to fall asleep [8]. The PFQ item regarding sleep was generalized, not specifying the type of impact of the low back pain on sleep in detail. Subjects simply answered with "true” or "false” to the statement "I don’t sleep well”. However, subjects in both treatment groups reported improvements in this category following initiation of treatment, and subjects treated with the combina- 
tion of diclofenac and B vitamins showed greater improvement after 3 days of treatment as compared to subjects treated with diclofenac monotherapy. This finding may be of use to clinical practice as it indicates that reduction in pain is associated with a positive impact on other aspects of the treated subject's life.

The combination of diclofenac with vitamins $\mathrm{B}_{1}, \mathrm{~B}_{6}$, and $\mathrm{B}_{12}$ has been reported to be beneficial in the treatment of painful conditions, including post-operative pain [9], osteoarthritis [10], and especially low back pain. Prior to the publication of the DOLOR trial, encouraging results in clinical studies of low back pain employing the combination of vitamins $\mathrm{B}_{1}, \mathrm{~B}_{6}$, and $\mathrm{B}_{12}$ with diclofenac had been reported in the literature, especially in terms of shortened treatment time to satisfactory analgesia when compared with diclofenac monotherapy [11]-[14]. The results of the DOLOR study confirmed these findings and the results of this post-hoc analysis reinforced the benefit of adding vitamins $B_{1}, B_{6}$, and $B_{12}$ to diclofenac therapy in terms of shorter treatment time to desired clinical effect.

\section{Conclusion}

Improvement in overall PFQ scores was observed in both treatment groups, though the specific items of the questionnaire of the subjects in group DB at Visit 2 showed greater improvement in areas related to sleep quality, mobility, ability to wash and dry, ability to walk distances, and posture comfort. These results serve to corroborate the previously published data of the DOLOR study, which indicates a benefit of combining the B vitamins with diclofenac in the treatment of patients with low back pain, yielding shorter treatment time to resolution of the lumbago in the treated patients.

\section{References}

[1] Mibielli, M.A., Geller, M., Cohen, J.C., Goldberg, S.G., Cohen, M.T., Nunes, C.P., et al. (2009) Diclofenac plus B Vitamins versus Diclofenac Monotherapy in Lumbago: The DOLOR Study. Current Medical Research and Opinion, 25, 2589-2599.

[2] Breivik, H., Callett, B., Ventafridda, V., Cohen, R. and Gallacher, D. (2006) Survey of Chronic Pain in Europe: Prevalence, Impact on Daily Life, and Treatment. European Journal of Pain, 10, 287-333. http://dx.doi.org/10.1016/j.ejpain.2005.06.009

[3] Manek, N. and MacGregor, A.J. (2005) Epidemiology of Low Back Disorders. Current Opinion in Rheumatology, 17, 134-140.

[4] Froud, R., Patterson, S., Eldridge, S., Seale, C., Pincus, T., Rajendran, D., et al. (2014) A Systematic Review and Meta-Synthesis of the Impact of Low Back Pain on People’s Lives. BMC Musculoskeletal Disorders, 15, 50. http://dx.doi.org/10.1186/1471-2474-15-50

[5] Hart, L.G., Deyo, R.A. and Cherkin, D.C. (1995) Physician Office Visits for Low Back Pain. Frequency, Clinical Evaluation, and Treatment Patterns from a U.S. National Survey. Spine, 20, 11-19. http://dx.doi.org/10.1097/00007632-199501000-00003

[6] Atlas, S.J. and Deyo, R.A. (2001) Evaluating and Managing Acute Low Back Pain in the Primary Care Setting. Journal of General Internal Medicine, 16, 120-131. http://dx.doi.org/10.1111/j.1525-1497.2001.91141.x

[7] Maetzel, A. and Li, L. (2002) The Economic Burden of Low Back Pain: A Review of Studies Published between 1996 and 2001. Best Practice \& Research Clinical Rheumatology, 16, 23-30. http://dx.doi.org/10.1053/berh.2001.0204

[8] Kelly, G.A., Blake, C., Power, C.K., O’keeffe, D. and Fullen, B.M. (2011) The Association between Chronic Low Back Pain and Sleep: A Systematic Review. Clinical Journal of Pain, 27, 169-181. http://dx.doi.org/10.1097/AJP.0b013e3181f3bdd5

[9] Perez-Flores, E., Medina-Santillán, R., Reyes-García, G. and Mateos-Garía, E. (2003) Combination of Diclofenac plus B Vitamins in Acute Pain after Tonsillectomy: A Pilot Study. Proceedings of the Western Pharmacology Society, 46, 88-90.

[10] Nunes, C.P., de Oliveira, P.C., de Oliveira, J.M., Mibielli, M.A., Cohen, J.C. and Nunes, F.P. (2005) A Double-Blind, Comparative, Placebo-Controlled Study in Two Arms of the Safety and Efficacy of the Anti-Inflammatory and Analgesic Action of the Association of Cyanocobalamin, Pyridoxine Chlorihydrate, Thiamine Mononitrate and Diclofenac Sodium in Tablets, in Patients with Osteoarthritis. Revista Brasileira de Medicina, 62, 486-491.

[11] Kuhlwein, A., Meyer, H.J. and Koehler, C.O. (1990) Reduced Need for Diclofenac with Concomitant B Vitamin Therapy: Results of a Double-Blind Clinical Study with Reduced Diclofenac-Dosage (75 mg Diclofenac vs. 75 mg Diclofenac plus B Vitamins Daily) in Patients with Acute Lumbago. Klinische Wochenschrift, 68, 107-115. http://dx.doi.org/10.1007/BF01646857 
[12] Vetter, G., Brüggemann, G., Lettko, M. (1988) Verkürzung der diclofenac-therapie durch B-vinamine. Ergebnisse einer randomisierten doppelblindstudie, diclofenac $50 \mathrm{mg}$ gegen diclofenac $50 \mathrm{mg}$ plus B-vitamine, bei schmerzhaften wirbelsäulenerkrankungen mit degenerativen veränderungen. Zeitschrift für Rheumatologie, 47, 351-362.

[13] Lettko, M. (1986) Ergebnisse einer Doppelblindstudie, Diclofenac und B-Vitamine gegen Diclofenac, zur Prüfung der additiven Wirksamkeit der B-Vitamine. Rheuma, Schmerz \& Entzündung, 8, 22-30.

[14] Brüggemann, G., Koehler, C.O. and Koch, E.M.W. (1990) Ergebnisse einer Doppelblindprüfung Diclofenac + Vitamin $\mathrm{B}_{1}, \mathrm{~B}_{6}, \mathrm{~B}_{12}$ versus Diclofenac bei Patienten mit akuten Beschwerden im Lendenwirbelsäulenbereich. Klinische Wochenschrift, 68, 116-120. http://dx.doi.org/10.1007/BF01646858 